\title{
Experiencias de enfermería ante la muerte neonatal: Una mirada fenomenológica
}

\author{
Nursing experiences in the event of neonatal death: A phenomenological view \\ María Cárdenas de Fernández ${ }^{1,3}$, Fátima Pinto Rojas ${ }^{2}$, Rosa Reina Caballero ${ }^{1}$, Anika Remuzgo Artezano ${ }^{3}$, \\ Gladys Jiménez Rodríguez².
}

\section{RESUMEN}

La muerte es una situación universal que todos los seres humanos vivimos a lo largo de nuestra vida. Es difícil de tratar y más afrontar la pérdida de un neonato. Esta situación muchas veces a la enfermera le hace tener sentimientos de culpa, de haber fallado. Abordar la muerte neonatal es una tarea delicada, lograr que el familiar exprese sus emociones, que drene su tristeza es muy difícil y más cuando está sumergido en su dolor. Dicha experiencia no deja indiferente a las enfermeras, que no saben cómo comportarse, ni cómo acompañar y cuidar a una mujer y a su pareja cuando han sufrido una pérdida. El objetivo es interpretar los significados de la experiencia vivida por los profesionales de enfermería en situaciones de muerte neonatal. Esta investigación tiene un abordaje fenomenológico hermenéutico, su paradigma es cualitativo. Para el análisis de los datos se utilizó las etapas de Herbert Spiegelberg. El estudio se realizó en el Hospital Universitario Ángel Larralde de Valencia, Estado Carabobo, Venezuela, en el Servicio de Neonatología. Se contó con tres profesionales de enfermería y dos madres que perdieron a su hijo, se realizó entrevista a profundidad, obteniendo categorías y subcategorías para posterior teorización. Como conclusión se obtiene que el profesional de enfermería necesita ser preparado ante situaciones de muerte y es por ello que cada momento del cuidado es único, y de la forma en que los mismos sean brindados así serán reflejados en el paciente y familia por muy dolorosa que esta sea.

Palabras clave: enfermería, muerte, neonato.

\begin{abstract}
Death is a universal situation that all human beings live throughout their lives, it is difficult to deal with and much more confront the loss of a newborn. This is a situation many times the nursing professional has feelings of guilt, have failed Addressing neonatal death is a delicate task to get the family member to express their emotions, to drain their sadness is very difficult and much more when immersed in their pain, this experience does not leave indifferent to the nursing professionals who do not know how to behave, nor how to accompany and care for a woman and her partner when they have suffered a loss. The objective is to interpret the meanings of the experience experienced by nursing professionals in situations of neonatal death. This research has a phenomenological hermeneutical approach, its paradigm is qualitative and the method used for this study was of Herbert Spiegelberg. The study was carried out at the Ángel Larralde University Hospital "Carabobo Hospital" of Valencia, State Carabobo, Venezuela in the Neonatology Service, it was attended by three nursing professionals and two mothers who lost their son, and an in-depth interview was conducted, obtaining subcategories for later theorization. As a conclusion it is obtained that the nursing professional needs to be prepared before death situations and that is why each moment of the care is unique, and the way in which they are provided will be reflected in the patient and family, however painful may occur.
\end{abstract}

Keywords: nursing, death, neonate.

${ }^{1}$ Universidad de Carabobo. Venezuela.

${ }^{2}$ Hospital Universitario Dr. Ángel Larralde. Venezuela.

${ }^{3}$ Universidad Norbert Wiener. Lima - Perú.

\section{INTRODUCCIÓN}

El profesional de enfermería, en la búsqueda continua del equilibrio, enfrenta incontables retos ya que su labor implica ayudar a personas sanas o enfermas para lograr su recuperación o una muerte tranquila. Esta última tiende a ser muy dolorosa, porque se preparan para servir a la persona 
enferma y lograr su bienestar, pero muy poco se preparan para el final o simplemente no quieren formar parte de ese momento. La muerte es considerada el fin de la vida. La muerte neonatal en las unidades de cuidados intensivos es entendida como el cese de todas las funciones vitales del cuerpo, incluyendo el latido del corazón, la actividad cerebral (incluido el tallo cerebral), y la respiración, en un niño entre su nacimiento y las primeras cuatro semanas de vida, independientemente de si la causa es prematurez, defectos congénitos, malformaciones, etc. (1).

Así, esta situación es inevitable en nuestra profesión porque se vive día a día la muerte de otra persona, la cual hace que el profesional de enfermería tenga sentimientos de culpa, de haber fallado, incluso de manera inconsciente nos apartamos de los padres que en ese instante se encuentran tristes por haber perdido a su hijo. A menudo no sabemos que decir y nos sentimos incapaces de ofrecer palabras de consuelo (2). El peso de los fallecimientos en menores de 28 días en la mortalidad infantil muestra diferencias al interior de Venezuela: en Cojedes y Trujillo se concentran más del $80 \%$ de los decesos, mientras que Delta Amacuro y Miranda son los estados con menor peso (con 58\% y 62\%). Las afecciones originadas en el periodo perinatal son la principal causa de mortalidad infantil con una proporción de $61,30 \%$, las anomalías congénitas acumularon $20,88 \%$ y los accidentes de todo tipo son la tercera causa de mortalidad con $3,72 \%$.(3).

La muerte de neonatal para el periodo 20142015 en el servicio de neonatología del hospital Carabobo ha disminuido considerablemente con un aproximado de tres neonatos que mueren por mes, por prematuros o sepsis neonatal. En comparación con otro estado como Táchira, al menos catorces niños recién nacidos han fallecido en los primeros treinta y sietes días del 2012, y en Maracay alrededor de $70 \%$ corresponde a muertes de neonatos (un promedio de 21 fallecimientos al mes de recién nacidos (4).

Como profesionales de enfermería, se sabe que todos los seres humanos mueren, pero de acuerdo a las creencias y pensamientos que tiene cada individuo como persona única que es, actúa más como persona, que como profesional de enfermería. Debido a esto uno piensa y reacciona de acuerdo a sus vivencias, y el suceso es más personal que profesional. Por eso la muerte de un neonato puede traerle muchos sentimientos, reacciones y actitudes que reflejan tristeza y se ven reflejados en algún familiar, un vecino o en su propio hijo, debido a que es una expresión propia. Porque uno desempeña otros roles como es ser madre, tía, esposa, ama de casa y es muy difícil desligarse de estas emociones. Carmona (5), en su investigación realizada en Venezuela titulada "Muerte, El duelo y el equipo de salud", trata sobre la muerte y el duelo, compañeros constantes e inevitables de la vida y sobre el equipo de salud, que en su día a día, se ven sumergidos en sus propios conflictos y envueltos en los conflictos del otro.

Durante la experiencia del profesional de enfermería, se observa que ante la pérdida de un neonato el equipo de salud, específicamente los profesionales de enfermería realizan muchas funciones que son parte de su labor, entre ellas los cuidados post mortem al recién nacido, siendo muy dolorosa esa situación y muchas veces prefiriendo no hacerla y menos estar ahí, tener que desconectarlo y prepararlo para que lo vean sus familiares, tratar de presentarlo de la manera menos dolorosa y traumática para ellos es muy difícil, pero al llegar los padres uno prefiere muchas veces alejarse de esa situación, no dicen y no hacen nada. Comentan que prefieren que hubiera muerto en otra guardia y no en las de ellas, se sienten incómodos, rogando que la guardia culmine rápido y se retiran a realizar otras actividades.

Estos lamentables hechos ocurren a nivel mundial, sin importar religión, cultura y situación económica, esto produce pena y tristeza a los padres y familiares de los neonatos que han fallecido (6). Lamentablemente en nuestro medio el equipo de salud ha sido formado para promover la salud, prolongar la vida, pero no para enfrentar dignamente la muerte y apoyarlo en sus decisiones. Esto no implica enfrentar problemas profesionales y técnicos sino también emocionales y espirituales.

Para comprender el fenómeno social del significado que tiene para los profesionales de enfermería sus vivencias sobre la muerte neonatal, se toma como punto de referencia el análisis del ser que hace Heidegger, la cual se constituye en la corriente psicológica derivada de la psiquiatría y la filosofía que permite comprender la existencia, sustentada en el sentido y la significación (7).

Todo lo anteriormente expuesto nos plantea una interrogante que puede resumir nuestra preocupación ante tal problemática: ¿Qué significado le otorga el profesional de enfermería a la experiencia vivida en situaciones de muerte neonatal? Esto para generar estrategias de actuación ante esta situación, que permitan ayudar al profesional de enfermería, padres y familiares a interpretar la vivencia del profesional de enfermería en situaciones de muerte neonatal.

Por todo lo expuesto, el objetivo de esta investigación fue interpretar las experiencias de 
enfermería ante la muerte neonatal desde la fenomenología.

\section{MATERIALES Y MÉTODOS}

En la búsqueda de la respuesta a la pregunta surgida de la temática en estudio, se decidió por un abordaje de tipo cualitativo. La matriz epistémica seleccionada fue la fenomenológicahermenéutica. El proceso de recolección de datos se hizo por medio de la entrevista a profundidad, las cuales fueron realizadas a través de visitas personalizadas en la que se estableció interrelación, fueron en total 5 visitas con una duración de 45 minutos, vale decir que las entrevistas fueron grabadas. La muestra estuvo conformada por tres profesionales de enfermería y dos madres que perdieron a sus hijos, quienes firmaron un consentimiento informado. Luego se desgrabaron los datos, se agruparon para formar las categorías que representan los significados individuales de cada una de las entrevistadas, a través de las 6 etapas de Spiegelberg.

\section{RESULTADOS Y DISCUSIÓN}

Para el análisis se escogió las etapas de Spiegelberg a través de las 6 etapas emergieron cuatro categorías que se presentan en la Tabla 1 .

Tabla 1: Matriz de categorización

\begin{tabular}{|l|l|l|}
\hline CATEGORÍAS & SUB-CATEGORÍAS & CÓDIGOS \\
\hline $\begin{array}{l}\text { Vivencia del profesional de } \\
\text { enfermería en situaciones de } \\
\text { muerte neonatal }\end{array}$ & $\begin{array}{l}\text { Relatando la experiencia } \\
\text { Significado de la muerte } \\
\text { neonatal }\end{array}$ & VLMNPE \\
\hline Sentimientos que emergieron & $\begin{array}{l}\text { Sentimiento de culpa } \\
\text { Sentimiento de tristeza y } \\
\text { compasión }\end{array}$ & SQE \\
\hline $\begin{array}{l}\text { Herramientas de apoyo para el } \\
\text { manejo de la muerte neonatal }\end{array}$ & $\begin{array}{l}\text { Apoyo emocional } \\
\text { Orientación de enfermería } \\
\text { Sección educativa }\end{array}$ & HDAPMMN \\
\hline Conceptualización de muerte & $\begin{array}{l}\text { Desde la perspectiva } \\
\text { religiosa y sus creencias }\end{array}$ & CDM \\
\hline
\end{tabular}

Tabla 2: Matriz de triangulación

\begin{tabular}{|c|c|c|c|}
\hline CATEGORÍAS & $\begin{array}{l}\text { INFORMANTES } \quad \text { CLAVES/ } \\
\text { INFORMANTES EXTERNOS } \\
\text { Enfermera1, } \quad \text { Enfermera2, } \\
\text { Enfermera3, Madre1, Madre2 }\end{array}$ & BIBLIOGRAFÍA & $\begin{array}{l}\text { INTERPRETACIÓN DEL } \\
\text { INVESTIGADOR }\end{array}$ \\
\hline $\begin{array}{l}\text { Categoría 1 } \\
\text { Vivenciando la } \\
\text { muerte neonatal } \\
\text { desde la perspectiva } \\
\text { de enfermería y la } \\
\text { madre }\end{array}$ & $\begin{array}{l}\text { E1. Una experiencia que tuve hace } \\
\text { como cinco años un bebe que estaba } \\
\text { relativamente estable y de repente } \\
\text { falleció. Fue impactante y fuerte era } \\
\text { un bebe a término. } \\
\text { E2. Fue una experiencia de muerte } \\
\text { neonatal más reciente fue el Rn xxx, } \\
\text { duro casi un mes y medio en la } \\
\text { unidad. fue muy fuerte porque el } \\
\text { niño lucho bastante } \\
\text { E3. La de un bebe que la mama se } \\
\text { controló todo el embarazo, tomo } \\
\text { todas sus vitaminas, pero durante el } \\
\text { parto tuvo complicaciones. no lo } \\
\text { supero y falleció fue impactante } \\
\text { M1. fue una experiencia inesperada, } \\
\text { muy impactante } \\
\text { M2. Una de la experiencia muy } \\
\text { fuerte que tuve con mi hija. } \\
\text { Obviamente jamás en mi vida lo voy } \\
\text { a olvidar. }\end{array}$ & $\begin{array}{lr}\text { Peplau (8) la salud } \\
\text { consta de condiciones } \\
\text { interpersonales } \\
\text { psicológicas } \\
\text { interactúan. } \\
\text { promovida a través del } \\
\text { proceso interpersonal. } \\
\text { Peplau, basa su modelo } \\
\text { en la enfermería } \\
\text { psicodinámica, que ella } \\
\text { define como la } \\
\text { utilización de la } \\
\text { comprensión de la } \\
\text { conducta de uno } \\
\text { mismo para ayudar a } \\
\text { los demás a identificar } \\
\text { sus } \\
\text { Dificultades }\end{array}$ & $\begin{array}{l}\text { La experiencia de la muerte } \\
\text { neonatal trae mucho impacto } \\
\text { a la persona que está pasando } \\
\text { por esa situación tener que } \\
\text { ver morir un ser tan pequeño } \\
\text { que tiene una vida por } \\
\text { delante no es nada fácil y son } \\
\text { vivencias que cada individuo } \\
\text { la expresa y vive de manera } \\
\text { única. }\end{array}$ \\
\hline
\end{tabular}




\begin{tabular}{|c|c|c|c|}
\hline $\begin{array}{l}\text { Categoría 2 } \\
\text { Sentimientos que } \\
\text { emergieron }\end{array}$ & $\begin{array}{l}\text { E1. Muchos sentimientos porque ala } \\
\text { vez sentimientos de culpa. de } \\
\text { tristeza porque no tuvo la } \\
\text { oportunidad de estar ese último } \\
\text { momento en contacto con su mama } \\
\text { y papa } \\
\text { E2. Mis sentimientos fue de mucho } \\
\text { dolor y compasión, lucho hasta el } \\
\text { final y no lo logro } \\
\text { E3. Impresión porque creemos que } \\
\text { estamos haciendo las cosas bien. } \\
\text { Todo tiene que cambiar que es un } \\
\text { propósito que solo DIOS sabe. } \\
\text { M1. Dolor, impotencia. } \\
\text { M2. Tristeza, dolor una sensación de } \\
\text { vacío bueno Dios verdad es grande y } \\
\text { él sabe porque hace las cosas y eso } \\
\text { fue lo yo pensé. }\end{array}$ & $\begin{array}{l}\text { Richard. S. Lazarus, } \\
\text { (9), sugiere la teoría de } \\
\text { incluir sentimiento en } \\
\text { el marco de las } \\
\text { emociones, ya que } \\
\text { éstas se conciben en } \\
\text { sentido muy amplio. Es } \\
\text { decir, considera } \\
\text { sentimiento y emoción } \\
\text { comor conceptos } \\
\text { interrelacionados, en el } \\
\text { cual el concepto } \\
\text { emoción englobaría al } \\
\text { sentimiento. Por ello } \\
\text { define sentimiento } \\
\text { como el componente } \\
\text { subjetivo o cognitivo } \\
\text { de las emociones }\end{array}$ & $\begin{array}{l}\text { En la categoría } 2 \text { se observa } \\
\text { que la muerte genera muchos } \\
\text { sentimientos, de tristeza, } \\
\text { dolor vacío, compasión } \\
\text { desde cualquier punto de } \\
\text { vista y que afecta a la } \\
\text { persona que está viviendo su } \\
\text { perdida y también a la que se } \\
\text { encuentra a su alrededor, } \\
\text { porque es una situación que } \\
\text { se espera en algún momento } \\
\text { de la vida pero que nunca } \\
\text { estamos preparados }\end{array}$ \\
\hline $\begin{array}{l}\text { Categoría 3 } \\
\text { Herramientas de } \\
\text { apoyo para el } \\
\text { manejo de la muerte } \\
\text { neonatal }\end{array}$ & $\begin{array}{l}\text { E1. Yo pienso que orientación a } \\
\text { enfermería es el punto clave. ayudar } \\
\text { a los papas para que puedan vivir su } \\
\text { duelo } \\
\text { E2. Apoyo por parte de los mismos } \\
\text { terapistas o psicólogos, cómo ayudar } \\
\text { a la familia, ya que la familia es } \\
\text { primordial aquí en el retén. } \\
\text { E3. estar con ella y darle unos 10, } \\
\text { min acompañarle darle palabras de } \\
\text { consuelo } \\
\text { M1. Tener compasión del otro, } \\
\text { dejarla tranquila } \\
\text { M2. Una palabra, a pesar del dolor } \\
\text { que uno siente en ese momento, un } \\
\text { abrazo eso para uno es bastante. }\end{array}$ & $\begin{array}{l}\text { El modelo de Peplau } \\
\text { (8) La interacción } \\
\text { enfermero-persona } \\
\text { usuaria constituye uno } \\
\text { de los ejes centrales de } \\
\text { la provisión del } \\
\text { cuidado de enfermería. } \\
\text { Interacción es el } \\
\text { proceso de contactar } \\
\text { con el otro, utilizando } \\
\text { como vehículo la } \\
\text { comunicación. Este } \\
\text { contacto ya sea a través } \\
\text { del cuerpo, las } \\
\text { palabras, los ojos, los } \\
\text { sonidos o los objetos } \\
\text { establece una relación. }\end{array}$ & $\begin{array}{l}\text { En esta categoría claramente } \\
\text { se observa que se necesita } \\
\text { ayuda para poder afrontar la } \\
\text { pérdida de un ser querido y } \\
\text { mucha preparación para el } \\
\text { profesional de enfermería } \\
\text { que vive diariamente la } \\
\text { muerte de otra persona, para } \\
\text { poder abordar esta situación } \\
\text { y ayudar a la madre, padre y } \\
\text { familia a canalizar su pérdida } \\
\text { de manera positiva y no } \\
\text { patológica. }\end{array}$ \\
\hline $\begin{array}{l}\text { Categoría } 4 \\
\text { Conceptualización } \\
\text { de la muerte }\end{array}$ & $\begin{array}{l}\text { E1. Es la pérdida física del ser } \\
\text { querido, perder algo dentro de ti } \\
\text { pero que es parte de la vida aunque } \\
\text { no entendamos porque las cosas } \\
\text { pasan, solo DIOS tiene el control, es } \\
\text { perder algo preciado para ti que } \\
\text { causa dolor, tristeza que a pesar el } \\
\text { tiempo aprendes a convivir con ello } \\
\text { pero no lo superas. } \\
\text { E2. Para mí la muerte es el paso a la } \\
\text { vida eterna en donde no habrá } \\
\text { dolencias, ni tristezas. Es el } \\
\text { descanso de la enfermedad. } \\
\text { E3. Es un proceso natural de la vida, } \\
\text { pero especialmente es una esperanza } \\
\text { de que algún momento nos } \\
\text { reencontraremos con ese ser } \\
\text { querido, también es una seguridad } \\
\text { de vida eterna más allá de lo natural. } \\
\text { M1. La muerte para mí es un estado } \\
\text { inesperado en la que estés, que todos } \\
\text { los seres humanos sabemos que } \\
\text { algún día vamos a fallecer pero no } \\
\text { estamos preparado para eso. } \\
\text { M2. La muerte para mí es un } \\
\text { fallecer, es pasar a otro mundo, en } \\
\text { algún momento estaré con mi hija } \\
\text { otra vez. }\end{array}$ & $\begin{array}{l}\text { La definición de } \\
\text { enfermería de } \\
\text { Henderson (10) tiene } \\
\text { como propósito medir } \\
\text { la eficiencia de la } \\
\text { enfermera al satisfacer } \\
\text { las necesidades del } \\
\text { paciente, por un lado y } \\
\text { por otro busca lograr } \\
\text { que el paciente con } \\
\text { enfermedades graves } \\
\text { alcance una muerte } \\
\text { tranquila, es el } \\
\text { mencionar el tema de la } \\
\text { muerte en su definición } \\
\text { lo que le da un toque } \\
\text { humanista. }\end{array}$ & $\begin{array}{l}\text { En esta categoría se aprecia } \\
\text { lo que refleja la muerte para } \\
\text { las personas que es el fin de } \\
\text { la vida, pero consideran que } \\
\text { hay un lugar donde no } \\
\text { sufrirán más, que es un } \\
\text { proceso doloroso que con la } \\
\text { ayuda de DIOS van a superar } \\
\text { y que recuperaran pero jamás } \\
\text { se olvidaran de sus seres } \\
\text { querido. }\end{array}$ \\
\hline
\end{tabular}




\section{CONSIDERACIONES FINALES}

La siguiente investigación me ha permitido descubrir la vivencia del profesional de enfermería y la madre en situaciones de muerte neonatal, como un proceso impactante el cual el profesional de enfermería necesita herramientas de apoyo para el manejo de la muerte, orientación, sección educativa y apoyo emocional de tal forma podrá ayudar a la familia, madre y padre en esa etapa tan difícil y ayudarlo a canalizar su duelo.

La Enfermería como disciplina orienta sus acciones al cuidado integral, es por ello que cada momento del cuidado es único, y de la forma en que los mismos sean brindados así serán reflejados en el paciente y su familia. Por ello que el personal a cargo del cuidado debe estar debidamente preparadas y así brindar una atención científica y humanizada para su paciente y sus familiares que están pasando por una situación de perdida.

\section{AUTOR DE CORRESPONDENCIA}

María Cárdenas de Fernandez

EP de Enfermería

Universidad Norbert Wiener

Dirección:

Teléfono:

\section{REFERENCIAS BIBLIOGRÁFICAS}

1. Aguinaga B, William, O." Enfermería, Muerte y Duelo. Un texto de reflexión académica" editorial Universidad Nacional de ColombiaBogotá 2010. 221.

2. Castañedo C, y cols. Capacitación y afrontamiento de las enfermeras de urgencias ante la muerte y el duelo .Enfermería Docente 2010, 91:16-18

3. Anuario de mortalidad, ministerio del poder popular para la salud septiembre https://www.ovsalud.org/descargas/publicacio nes/documentos-oficiales/AnuarioMortalidad-2012.pdf

4. Álvarez G, estadísticas de muerte neonatal, Hospital Carabobo servicio de epidemiologia 2014,2015

5. Carmona, Z. La muerte, el duelo y el equipo de salud. Revista de Salud Pública Valencia, Venezuela. 2008. P. 14-23.

6. Arraz, B, y cols. Intervención emocional en cuidados paliativos. Modelo y Protocolos. Barcelona- España: Ariel Ciencias Medica. 2014. P. 220
7. Heidegger, M. El Ser y el Tiempo. Traductor José Gaos, 3era Reimpresión. Buenos Aires: Argentina: Fondo de Cultura Económica. 1991

8. Barbara J. Callaway. Hildegard Peplau: psychiatric nurse of the century. 2002. ISBN 0-8261-3882-9

9. Richard Lazarus. Revista Latinoamericana de Psicología. Revista Latinoamericana de Psicología ISSN: 0120-0534 Vol: 37, N ${ }^{\circ} 1-2$ direccion.rlp@konradlorenz.edu.co

Fundación Universitaria Konrad Lorenz Colombia. 2005

10. Henderson, V. [Internet]. [Acceso 19 mar.2009]. Disponible en: http://www.ulpgc.es/descargadirecta.php 